\title{
CARRIER MEDIATED TRANSPORT OF AMINO ACIDS, SMALL PEPTIDES, AND THEIR DRUG ANALOGS*
}

\author{
Patrick J. Sinko, Ming Hu and Gordon L. Amidon \\ College of Pharmacy, University of Michigan, Ann Arbor, MI $48109-1065$ (U.S.A.)
}

The oral absorption of amino acids, small peptides and their analogs has been studied in rats using single pass intestinal perfusion. The experimental data was analyzed using a modified boundary layer method permitting the calculation of intrinsic membrane absorption parameters. Cefatrizine, L-Dopa, $\alpha$-methyldopa, and L-PHE demonstrated concentration dependent absorption. Furthermore, the absorption of the amino acid analog, L-Dopa, was significantly inhibited by L-Leucine. Cefatrizine absorption was significantly inhibited by the peptide PHE-PHE (20 $\mathrm{mM})$ whereas the amino acid $\mathrm{L}-\mathrm{PHE}(100 \mathrm{mM})$ did not inhibit its absorption. The results clearly demonstrate that $\mathrm{L}-D o p a$ and $\alpha$-methyldopa oral absorption occurs via the amino acid pathway, while cefatrizine absorption occurs via the peptide pathway. These results indicate that nutrient pathways may function as a possible route for the oral delivery of selected drugs.

\section{INTRODUCTION}

Most drugs are absorbed by a passive diffusion process where the fraction of dose absorbed is a function of the dose to solubility ratio, particle size, the partition coefficient, $\mathrm{p} K_{\mathrm{a}}$, stability, etc. In addition the absorption process is also molecular weight dependent $[1,2]$. Compounds with molecular weights of approximately 500 daltons and above are poorly absorbed from the gastrointestinal (GI) tract [3]. The low permeability of the GI mucosa for high molecular weight compounds may be attributable to the glycoprotein mucin that covers the absorptive surface area of the small intestine. Two mechanisms may account for the decrease in drug absorption. The gel-like mucin can act as physical barrier (low drug diffusivity) to high molecular weight compounds.

*Paper presented at the Third International Symposium on Recent Advances in Drug Delivery Systems, February 24-27, 1987, Salt Lake City, UT, U.S.A.
Additionally, ionic interactions with the glycoprotein may also lead to low drug diffusivity and hence, decrease the fraction of dose absorbed [4]. For example, Gibaldi [5] reported that intestinal mucin forms a nonabsorbable complex with streptomycin whereas Niibuchi et al. [4] concluded that the binding of gentamycin to mucin may account for the compound's poor absorption.

On the other hand, the transport of polar nutrients, such as the amino acids and di- or tripeptides, occurs by nonpassive mechanisms in the human small intestine [6-8]. The uptake of amino acids, for example, from the intestinal lumen is well characterized with saturable systems both in the brush border and basolateral membranes $[9,10]$. The peptide transport system, on the other hand, is not as well characterized but results suggest that peptides with greater than three amino acid residues are not ordinarily substrates for the peptide carrier but rather are substrates for brush border and 
luminal peptidases [8]. This suggests that drugs which are analogs of amino acids or small peptides may be absorbed by this nonpassive pathway which may then offer a unique route for oral delivery of peptides and peptide analogs.

Levodopa, an aromatic amino acid, and $\alpha$ methyldopa, the related $\alpha$-methyl analog, are structural analogs of the amino acids. In early studies of $\alpha$-methyldopa in rats [11], it was shown that absorption was concentration and $\mathrm{pH}$ dependent and is similar to amino acid absorption. For example, Amidon et al. [11] reported that the $\mathrm{pH}$ dependency of $\alpha$-methyldopa absorption is similar to leucine absorption in acidic solutions. Additionally, Au et al. [12] reported that selective absorption of the L-isomer of $\alpha$-methyldopa occurs in both humans and rats similar to amino acid absorption.

The $\beta$-lactam antibiotics are structurally analogous to cyclic tripeptides possessing a backbone that is similar to, for example, GLYALA-ALA. Early work by Quay and Foster [13] indicated the active transport of cephalexin in rat jejunum. More recently, several studies [14-16] suggest that the $\beta$-lactam antibiotics are absorbed by a carrier mediated pathway. However, some of the results are conflicting and a method of relating carrier mediated and passive absorption is still lacking. In this report the results of several recent studies on $\alpha$-methyldopa, L-Dopa, and cefatrizine are presented and compared with those for nutrient, amino acid and peptide absorption using a recently developed boundary layer approach to perfusion experiments [17].

\section{MATERIALS AND METHODS}

All experiments were performed on male Charles River rats, $250-400 \mathrm{~g}$, age 60 to 80 days. The rats were fasted overnight, twelve to eighteen hours before each experiment. Water was given ad libitum. Anesthesia was induced by an intramuscular injection of urethane $(50 \% \mathrm{w} / \mathrm{v}$, $1.5 \mathrm{~g} / \mathrm{kg}$ ). The rats were kept on a slide warmer
(GCA Corp., Chicago, IL) and under a heating lamp to maintain their normal body temperature throughout the experiment. The abdominal cavity was opened by a mid-line incision of three to four centimeters. The jejunum was located and cannulated at two to four centimeters below the ligament of Treitz and 6 to 15 centimeters distal to the first incision. The cannulae were secured with surgical silk sutures (40 Chromic Gut, Ethicon Inc., Somerville, N.J. ).

All tubing and cannulae were made of poly (tetrafluoroethylene) $\left(\right.$ Teflon $\left.^{\circledR}\right)$. Care was taken to maintain the inlet and outlet cannulae at the same height to avoid gravitational flow.

The perfusion solution consisted of an isoosmotic buffer, $0.01 \%$ (w/v) PEG 4000 with a tracer amount of its ${ }^{14} \mathrm{C}$ isotope, and the test compound. A phosphate buffer was used for the $\alpha$-methyldopa, L-Dopa, PHE, and PHE-PHE perfusions. A citric acid dibasic sodium phosphate buffer $(\mathrm{pH} 6.5)$ was used for the $\beta$-Lactam perfusions. The perfusate $(300 \pm 5$ $\mathrm{mOsm} / \mathrm{kg}$ water ) was maintained at $37^{\circ} \mathrm{C}$ by a water bath (Tek-Pro, American Dade, Miami, FL ). The osmolality was measured using either a model 5002 automatic osmometer (Precision Systems, Inc., Sudsbury, MA) or a model 5500 Vapor Pressure osmometer (Wescor Inc., Logan, UT).

The jejunal segment was perfused using a constant infusion pump (Harvard Apparatus, Model 931, S. Natick, MA) for 90 to 105 minutes. The perfusion flow rate was varied from 0.124 to $0.764 \mathrm{ml}$ per minute. Steady state was achieved in approximately 30 minutes after which four to six samples were taken at 10 to 15 minute intervals. All $\beta$-Lactam samples were simultaneously quenched at the experiments end with $6 \% \mathrm{w} / \mathrm{v}$ citric acid solution. After the last sample was taken, the length was measured by placing a piece of string along the intestine and measuring the string with a ruler.

\section{Analytical methods}

The samples were analyzed by liquid scintillation counting and by high performance liquid 
chromatography (HPLC). Samples were counted using a Beckman LS-9000 counter (Beckman Instruments Inc., Fullerton, CA) with automatic quench correction. A $0.5 \mathrm{ml}$ sample was mixed with $15 \mathrm{ml}$ of scintillation fluid (Ready-Solv, $\mathrm{Hp} / \mathrm{b}$, Beckman Instruments Inc.) before counting. Samples were counted using either a single or double channel technique for the amino acid analogs, $\beta$-lactams or PHE-PHE and L-PHE, respectively. The HPLC instrumentation consisted of a pump (Beckman 112 solvent delivery module, Beckman Instruments, Inc., Berkeley, CA), Waters Intelligent Sample Processor (WISP, model 710B, Waters Associates, Milford, MA), Ultraviolet detector (Kratos Spectroflow 783 absorbance detector, Kratos Analytical Instruments, Ramsey, $\mathrm{NJ}$ ), a reverse phase column ( $\mu$-Bondapak $\mathrm{C}_{18}$, Waters Associates, Milford, MA) for L-PHE, PHE-PHE, and cefatrizine, and a strong cation exchange column for L-Dopa and $\alpha$-methyldopa. The mobile phase for the $\beta$ lactam studies consisted of methanol and pH 5 citric acid-dibasic sodium phosphate buffer (22:78). Samples eluted at a flow rate of 1.5 $\mathrm{ml} / \mathrm{min}$. The mobile phase for L-Dopa and $\alpha$ methyldopa consisted of acetonitrile and $\mathrm{pH} 2.6$ ammonium phosphate buffer $(0.01 M)$ in the ratios 52:48 and 50:50, respectively. Samples eluted at a flow rate of 1.5 and $1.0 \mathrm{ml}$ per minute, respectively.

\section{THEORY}

\section{Water transport}

Water transport was monitored by comparing the total radioactivity of the inlet and outlet samples. ${ }^{14} \mathrm{C}$-Polyethylene glycol 4000 was used as the nonabsorbable marker. The percentage of water transport per centimeter length perfused for each sample was calculated from:

$$
\begin{aligned}
{\left[\left(A_{\mathrm{i}}-A_{\mathrm{f}}\right) / A_{\mathrm{f}}\right] } & \times 100 / \text { length }(\mathrm{cm}) \\
= & \% \text { Water transport } / \mathrm{cm}
\end{aligned}
$$

where $A_{\mathrm{i}}$ and $A_{\mathrm{f}}$ were the initial and final counts per minute of ${ }^{14} \mathrm{C}$-polyethylene glycol 4000 . Water transport below $0.5 \% / \mathrm{cm}$ of intestinal length is considered normal and experiments with water transport $>0.5 \% / \mathrm{cm}$ were not used in the determination of the kinetic parameters. The outlet concentrations were corrected for this volume change using eqn. (1).

\section{Estimation of unbiased membrane parameters}

Unbiased membrane parameters are estimated using a modified boundary layer model recently developed by Johnson and Amidon [17]. Briefly, the dimensionless effectively wall permeability, $P^{*}$ eff, is calculated from steadystate perfusion results:

$P^{*}{ }_{\text {eff }}=\left(1-C_{\mathrm{m}} / C_{0}\right) / 4 G z$

where $C_{0}$ and $C_{\mathrm{m}}$ are the inlet and outlet perfusate concentrations, respectively, and the Graetz number, $G z$, is

$G z=\pi D L /(2 Q)$

where $D$ is an estimate of the aqueous diffusion coefficient of a solute in the perfusate, $L$ is the length of the intestine, and $Q$ is the fluid flow rate. The aqueous permeability, $P_{\text {aq }}{ }^{*}$, can be estimated from:

$$
P_{\text {aq }}^{*-1}=A \cdot G z^{1 / 3}
$$

where $A$ is a constant and is calculated from a film model approximation to the boundary layer result:

$$
\begin{array}{ll}
A=10.00 G z+1.01 & \text { if } 0.004 \leq G z<0.01 \\
A=4.50 G z+1.065 & \text { if } 0.01 \leq G z<0.03 \\
A=2.25 G z+1.125 & \text { if } 0.03 \leq G z
\end{array}
$$

The concentration at the intestinal wall, $C_{u}$, and intrinsic wall permeability, $P^{*}{ }_{\mathrm{w}}$, are then calculated as follows:

$C_{\mathrm{w}}=C_{0}\left(1-P^{*}{ }_{\text {eff }} / P^{*}{ }_{\text {aq }}\right)$ 
and

$P^{*}{ }_{\mathrm{w}}=P^{*}{ }_{\mathrm{eff}} /\left(1-P^{*}{ }_{\mathrm{eff}} / P^{*}{ }_{\mathrm{aq}}\right)$

$P^{*}$ is a dimensionless permeability where $P^{*}=P$ $R / D . R$ is the radius of the intestine and $D$ is the estimated aqueous diffusion coefficient.

For a combination of carrier and passive uptake mechanisms:

$P^{*}{ }_{\mathrm{w}}=\frac{J^{*}{ }_{\max }}{K_{\mathrm{m}}+C_{\mathrm{w}}}+P^{*}{ }_{\mathrm{m}}$

where $J^{*}{ }_{\text {max }}$ is the maximal flux, $K_{\mathrm{m}}$ is the intrinsic Michaelis-Menten constant, and $P^{*}{ }_{m}$ is the intrinsic passive membrane permeability. The carrier permeability, which is defined as the limiting first order wall permeability can be defined as:

$P^{*}{ }_{\mathrm{c}}=J^{*}{ }_{\max } / K_{\mathrm{m}}$

Equation (7) becomes:

$P^{*}{ }_{\mathrm{w}}=P_{\mathrm{c}}^{*}\left(1+\left(C_{\mathrm{w}} / K_{\mathrm{m}}\right)\right)^{-1}+P^{*}{ }_{\mathrm{m}}$

This form of the wall permeability expression focuses on the parameters of particular interest in oral drug delivery and will be discussed in the subsequent section.

\section{RESULTS AND DISCUSSION}

The experimental data were fit to eqn. (7) or (9) using weighted nonlinear regression. The variance of $P_{\mathrm{w}}^{*}$ was not homogeneous over the concentration range studied, therefore, the data were weighted by the reciprocal variance of $P_{\mathrm{w}}^{*}$. All data were initially fit to the three parameter model, however, when the value of $P_{\mathrm{m}}^{*}$ was not significantly different from zero the model was reduced accordingly. The experimental data and fitted curves are shown for L-phenylalanine in Fig. 1, $\alpha$-methyldopa and cefatrizine in Fig. 2, and L-Dopa in Fig. 3. A summary of the intrinsic membrane parameters is listed in Table 1.

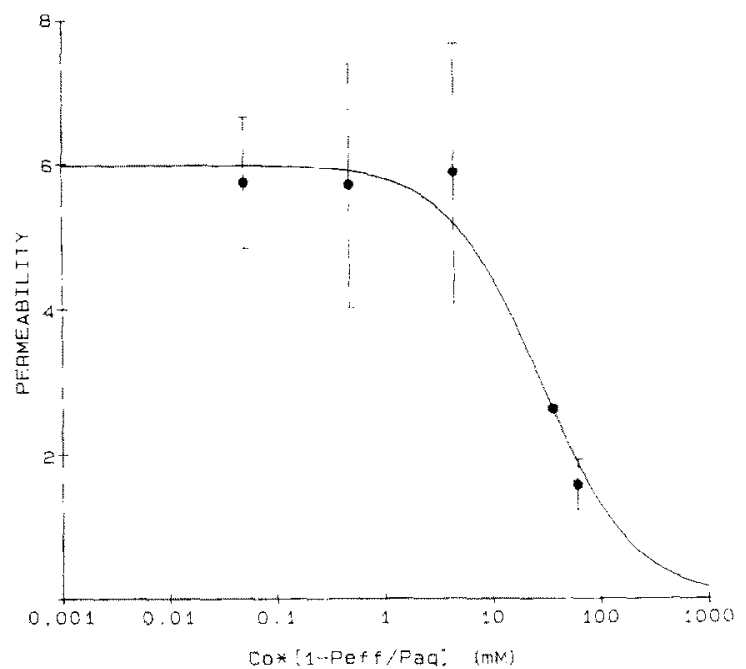

Fig. 1. The intrinsic wall permeability of L-PHE is plotted as a function of perfusate concentration corrected for the aqueous resistance. The intrinsic membrane absorption parameters are derived from nonlinear regression analysis on the data.

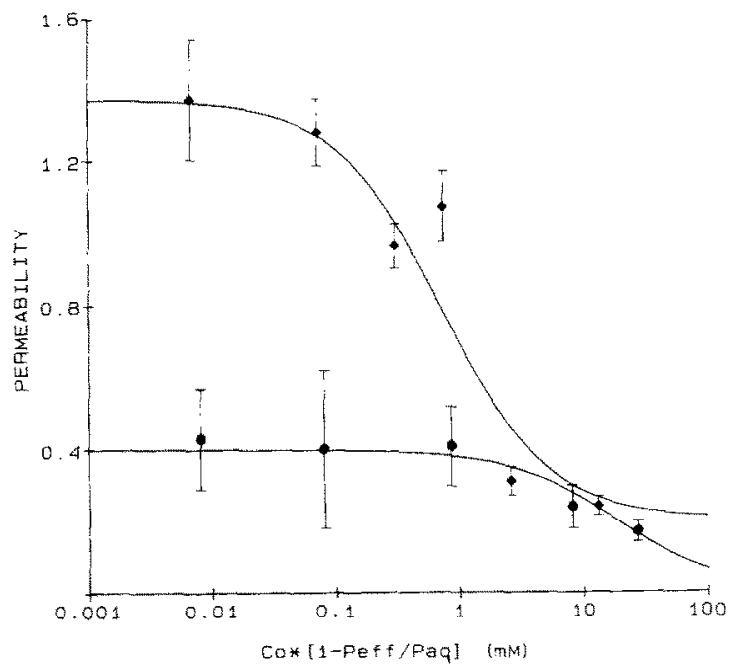

Fig. 2. The intrinsic wall permeability of $\alpha$-methyldopa ( $\bullet$ and cefatrizine $(\$)$ is plotted as a function of perfusate concentration corrected for the aqueous resistance. The intrinsic membrane absorption parameters are derived from nonlinear regression analysis on the data. 
TABLE 1

Summary of intrinsic membrane parameters ( \pm standard deviation)

\begin{tabular}{lllll}
\hline Compound & $J^{*}{ }_{\max }$ & $K_{\mathrm{m}}$ & $P^{*}{ }_{\mathrm{m}}$ & $P^{*}{ }_{\mathrm{c}}$ \\
\hline PHE & $49.9(3.6)$ & $5.3(1.4)$ & 0 & $6.0(0.7)$ \\
PHE-PHE & - & - & - & $6^{\mathrm{a}}$ \\
L-Dopa & - & - & - & $6^{\mathrm{a}}$ \\
$\alpha$-Methyldopa & $7.4(1.1)$ & $18.6(4.0)$ & - & $0.40(0.04)$ \\
Cefatrizine & $0.73(0.19)$ & $0.58(0.17)$ & $0.17(0.03)$ & $1.25(0.10)$
\end{tabular}

"Estimate of $P^{*}$..

\section{Amino acids and amino acid analogs}

Four independent carriers have been ascribed to amino acid transport in humans [18]. These include one for $\alpha$-amino-monoacidic amino acids (also known as neutral amino acids), one for basic amino acids, one for acidic amino acids, and one for amino acids with the amino group at the $\beta$ and $\delta$ position. The effect of side chain substitution on amino acid absorption, however, has not been extensively studied. Methyl substitution has had variable effects on amino acid absorption depending on the location of the substitution. Addition of a methyl group to the side chain of alanine, valine, leucine, and isoleucine increases the apparent affinity for the carrier [19], whereas replacement of the $\alpha$ hydrogen, as in $\alpha$-aminobutyric acid, reduces the affinity and extent of absorption. The ten fold decrease between the carrier permeability of L-dopa and $\alpha$-methyldopa may be attributable to the methyl substitution and would account for the decrease in the extent of absorption of $\alpha$-methyldopa. Results of competitive absorption studies performed with Ldopa and L-leucine at various concentrations are plotted in Fig. 3 . The high permeability and solubility limitation for L-Dopa prevent the determination of its $K_{\mathrm{m}}$. Given low doses of LDopa and $\alpha$-methyldopa, the largest concentration in the GI tract would likely be below the $K_{\mathrm{m}}$, in the one to five millimolar range. The wall permeability of L-Dopa in the first order region, at $0.1 \mathrm{mM}$ for example, can be used as an estimate of $P^{*}$. Therefore, as seen in Fig. 3, the

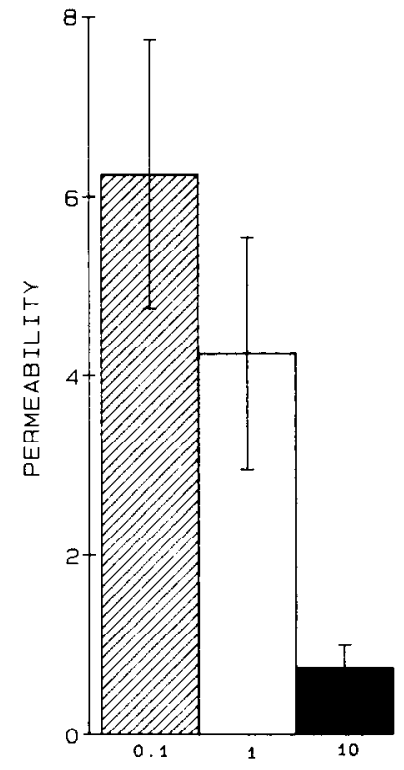

Fig. 3. The intrinsic wall permeability of L-Dopa is plotted as a function of perfusate concentration. The concentration of L-Dopa was $0.1 \mathrm{mM}(\mathbb{W}), 1 \mathrm{mM}(\square)$, and $10 \mathrm{mM}$ (a).

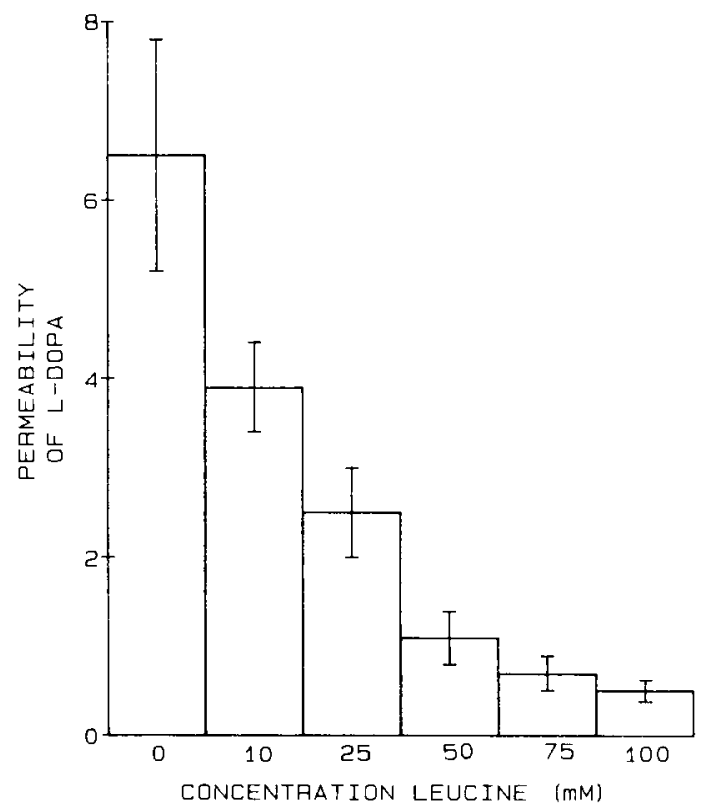

Fig. 4. Plot of the effect of the inhibitor L-Leucine on the wall permeability of L-Dopa. The concentration of L-Dopa remained constant at $0.1 \mathrm{mM}$ whereas the concentration of L-leucine varied from $0 \mathrm{mM}$ to $100 \mathrm{mM}$. 
carrier permeability of L-Dopa is approximately equal to 6 .

Studies using L-leucine as an inhibitor to LDopa (Fig. 4) clearly demonstrate competitive absorption confirming transport by the neutral amino acid carrier. Given the results in Table 1 , the additional hydroxyl groups on L-Dopa clearly have little effect on the carrier permeability since $P^{*}$ is similar to that for L-phenylalanine. It is also clear that the $\alpha$-methyl group has a profound effect on absorption of the amino acid analogs tested consistent with previous findings for amino acids [19].

\section{Small peptides and peptide analogs}

A fundamental difference between the peptide carrier and the amino acid carrier is its broad specificity in transporting peptide substrates [20-22], although the existence of more than one peptide carrier can not be ruled out. The results of cefatrizine perfusions are listed in Table 1 . However, the concentration range of PHE-PHE that could be studied was limited by the dipeptide's low aqueous solubility. Therefore, $P^{*}{ }_{c}$ was estimated from the intrinsic membrane permeability at low concentrations as for L-Dopa and is reported in Table 1. Furthermore, luminal and brush border hydrolysis of PHE-PHE complicates the analysis of peptide perfusions results. From the results in Table 1 , it is seen that PHE-PHE and L-PHE have nearly equal permeabilities. Given this result, the fact di- or tri-peptides are absorbed intact across the mucosal brush border [23], that only free amino acids reach the portal circulation [8], and that the net flux of L-PHE is doubled when PHE-PHE is absorbed, it can be seen that PHE absorption is more efficient from the peptide PHE-PHE then from the amino acid. These results agree with previous studies [24] in humans using the amino acids GLY and GLYGLY.

The results for cefatrizine demonstrate that it is much less permeable than PHE-PHE but is more permeable than $\alpha$-methyldopa (Table 1). Co-perfusion of cefatrizine $(0.1 \mathrm{mM})$ and the dipeptide PHE-GLY $(20 \mathrm{~m} M)$ resulted in a

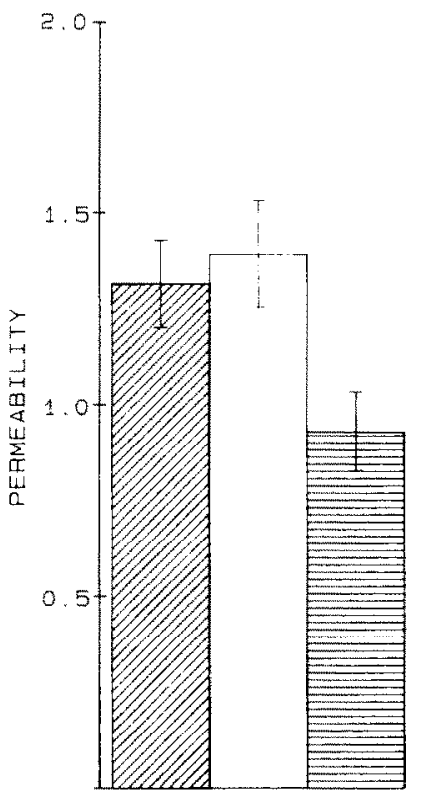

Fig. 5. Plot of the effect of amino acids and peptides on the wall permeability of cefatrizine $(\mathbb{Z})$, cefatrizine in the presence of $L$-Phenylalanine $(\square)$, or Phenylalanyl-Glycine ( The concentration of cefatrizine remained constant at $0.01 \mathrm{mM}$ while the concentration of L-PHE and PHE-GLY was $100 \mathrm{mM}$, and $20 \mathrm{mM}$, respectively.

significant decrease in cefatrizine permeability whereas the amino acid L-phenylalanine (100 $\mathrm{m} M$ ) did not inhibit cefatrizine absorption (Fig. 5). These results are consistent with the hypothesis that cefatrizine transport occurs by the peptide pathway.

\section{CONCLUSION}

In summary, these results clearly demonstrate that L-Dopa and $\alpha$-methyldopa oral absorption occurs via the amino acid pathway, while cefatrizine absorption occurs via the peptide pathway. The effect of $\alpha$-methyl substitution on the absorption of $\alpha$-methyldopa is dramatic and further supports a carrier pathway for absorption since a passive absorption mechanism would suggest that $\alpha$-methyldopa would be more membrane permeable. On the other hand, the peptide pathway may be less structurally specific since cefatrizine is absorbed reasonably well via the peptide pathway. 


\section{ACKNOWLEDGEMENT}

This work was in part supported by NIH Grant No. 1ROGM37188 and the SmithKline Beckman Corporation.

\section{REFERENCES}

1 V.S. Chadwick, S.F. Phillips and A.F. Hoffman, Measurements of intestinal permeability using lw molecular weight polyethylene glycols (PEG 400). I. Chemical analysis and biological properties of $P E G$ 400, Gastroenterology, 73 (1977) 241-246.

2 V.S. Chadwick, S.F. Phillips and A.F. Hoffman, Measurements of intestinal permeability using low molecular weight polyethylene glycols (PEG 400). II. Application to normal and abnormal permeability states in man and animals, Gastroenterology, 73 (1977) 247-251.

3 C. Tagesson, P.A. Andersson, T. Andersson, T. Bolin, M. Kallberg and R. Sjodahl, Passage of molecules through the wall of the gastrointestinal tract; measurement of intestinal permeability to polyethylene glycols in the 634-1338 dalton range (PEG 1000), Scand. J.Gastroenterol., 18 (1983) 481-486.

4 J. Niibuchi, Y. Aramaki and S. Tsuchiya, Binding of antibiotics to rat intestinal mucin, Int. J. Pharm., 30 (1986) 181-187.

5 M. Gibaldi, Introduction of Biopharmaceutics, Lee and Febiger, Philadelphia, 1971, p. 16.

6 G. Wiseman, Absorption of amino acids, in: W. Heidel (Ed.), Handbook of Physiology, Am. Physiol. Soc., Washington, D.C., 1968, pp. 1277-1307.

7 G. Wiseman, Absorption of protein digestion products, in: D.H. Smyth (Ed.) , Biomembranes 4A, Intestinal Absorption, Plenum Press, London, 1974, pp. 363-481.

8 S.A. Adibi and Y.S. Kim, Peptide absorption and hydrolysis, in: L.R. Johnson (Ed.), Physiology of the Gastrointestinal Tract, Raven Press, New York, 1981, pp. 1073-1095.

9 G. Danisi, Y.H. Tai and P.F. Curran, Mucosal and serosal fluxes of alanine in rabbit ileum, Biochim. Biophys. Acta, 455 (1976) 200-213.

10 J.J. Hajjar and P.F. Curran, Characteristics of the amino acid transport system in the mucosal border of rabbit ileum, J. Gen. Physiol., 56 (1970) 673-691.

11 G.L. Amidon, A.E. Merfield and J.B. Dressman, Con- centration and $\mathrm{pH}$ dependency of $\alpha$-methyldopa ahsorption in rat intestine, J. Pharm. Pharmacol., 38 (1986) 363-368.

12 W.Y.W. Au, L.G. Dring, D.G. Grahame-Smith, P. Isaac and R.T. Williams, The metabolism of ${ }^{14} \mathrm{C}$-labelled $\alpha$ methyldopa in normal and hypertensive human subjects, Biochem. J., 129 (1972) 1-10.

13 J.F. Quay and L. Foster, Cephalexin penetration of the surviving rat intestine, Physiologist, 13 (1970) 287-290.

14 T. Kimura, T. Yamamoto, M. Mizuno, Y. Suga, S. Kitade and $\mathrm{H}$. Sezaki, Characterization of aminocephalosporin transport across rat small intestine, J. Pharm. Dyn., 6 (1983) 246-253.

15 T. Kimura, H. Endo, M. Yoshikawa, S. Muranishi and H. Sezaki, Carrier mediated transport systems for aminopenicillins in rat small intestine, J. Pharm. Dyn., 1 (1978) 262-267.

16 E. Nakashima, A. Tsuji, S. Kagatani and T. Yamana, Intestinal absorption mechanism of amino- $\beta$-lactam antibiotics. III. Kinetics of carrier-mediated transport across the rat small intestine in situ, J. Pharm. Dyn., 7 (1984) 452-464.

17 D.A. Johnson and G.L. Amidon, Estimating intrinsic membrane transport parameters from perfused intestine experiments: A boundary layer approach to estimating the aqueous resistance, J. Theor. Biol., in press.

18 B.G. Munck, Intestinal absorption of amino acids, in: L.R. Johnson (Ed.), Physiology of the Gastrointestinal Tract, Raven Press, New York, pp. 1097-1122.

19 R.L. Preston, J.F. Schaeffer and P.F. Curran, Struc ture affinity relationships of substrates for the neutral amino acid transport system in rabbit ileum, J. Gen. Physiol., 64 (1974) 443-467.

20 M. Das and A.N. Radhakrishnan, Studies on a wide spectrum intestinal dipeptide uptake system in the monkey and in the human, Biochem. J., 146 (1975) 133-139.

21 D.M. Matthews, R.H. Gandy, E. Taylor, and D. Burston, Influx of two dipeptides, glycylsarcosine and Lglutamic acid, into hamster jejunum in vitro, Clin. Sci., 56 (1979) 15-23.

22 E. Taylor, D. Burston, and D.M. Matthews, Influx of glycylsarcosine and L-lysyl-L-lysine into hamster jejunum in vitro, Clin. Sci., 58 (1980) 221-225.

23 D.M. Matthews, Intestinal absorption of peptides, Physiol. Rev., 55 (1975) 537-608.

24 S.A. Adibi, Intestinal transport of dipeptides in man: Relative importance of hydrolysis and intact absorption. J. Clin. Invest., 50 (1971) 2266-2275. 